1 MSk Lab, Imperial College London, London W12 OBZ, UK

2 Department of Primary Care and Public Health, Imperial College London

3 Hull-York Medical School, York, UK

4 Imperial College Healthcare NHS Trust, London

5 HQ Army Medical Services, Robertson House, Camberley, UK

Correspondence to: D Salman d.salman11@imperial.ac.uk Cite this as: BMJ 2021;372:m4721 http://dx.doi.org/10.1136/bmj.m4721 Published: 08 January 2021

PRACTICE POINTER

\title{
Returning to physical activity after covid-19
}

\author{
David Salman, ${ }^{1,2}$ Dane Vishnubala, ${ }^{2,3}$ Peter Le Feuvre, ${ }^{1,5}$ Thomas Beaney, ${ }^{2}$ Jonathan Korgaonkar, ${ }^{4}$
} Azeem Majeed, ${ }^{2}$ Alison H McGregor

\section{What you need to know}

- Risk stratify patients before recommending a return to physical activity in people who have had covid-19. Patients with ongoing symptoms or who had severe covid-19 or a history suggestive of cardiac involvement need further clinical assessment

- Only return to exercise after at least seven days free of symptoms, and begin with at least two weeks of minimal exertion

- Use daily self monitoring to track progress, including when to seek further help

Our professional experience suggests that, after mild suspected covid-19, a proportion of people experience a prolonged recovery, particularly when trying to return to exercise. Moreover, there is increasing recognition of potential long term complications of covid-19, including enduring illness ("post-acute" or "long” covid), cardiopulmonary disease, and psychological sequelae in some people. ${ }^{1-4}$ This article offers a pragmatic approach to help patients safely return to physical activity after symptomatic SARS-CoV-2 infection, focusing on those who have lost fitness or had a prolonged period of inactivity but who do not have an enduring post-acute covid-19 illness. It is based on current evidence and consensus statements, and our own multidisciplinary experience in sports and exercise medicine, rehabilitation, and primary care.

The health benefits of being physically active, from cardiovascular to mental health, are well established. ${ }^{5}$ Conversely, the harms of physical inactivity mean it is a major risk factor for non-communicable disease worldwide, alongside others such as cigarette smoking or obesity. ${ }^{7}$ Before the covid-19 pandemic, over a third of people in the UK were not physically active enough for good health. ${ }^{8}$ There is evidence of a further decline in physical activity since the start of the pandemic for people with chronic conditions such as obesity and hypertension ${ }^{9}$; conditions associated with worse outcomes from covid-19. ${ }^{10}$ Brief advice in primary care can help people to take up physical activity, with the associated lifelong positive health impacts, and help those recovering from illness to return to previous levels of physical activity or beyond. ${ }^{11}$ People may feel unsure of how and when to return to physical activity after covid-19, and whether it is safe. Some may have tried to return to their baseline exercise, and found they were unable to do so, causing concern ("When will I get back to normal”?). Consensus statements published to date have focused on athletes, discussing how and when to return to sporting activity after covid-19. ${ }^{1213}$ However, physical activity (any movement of the body where energy is expended) encompasses much more than sport, and is, or should be, part of everyday life. UK public health guidance is for 150 minutes of moderate intensity physical activity (where there is moderate effort, the rate of breathing increases but it is easy to talk) or 75 minutes of vigorous intensity physical activity (the rate of breathing increases and it is difficult to talk) per week, in addition to muscle strengthening activities on at least two days each week. ${ }^{6}$ Although this can include exercise (planned and structured physical activity done with the intention of improving physical fitness ${ }^{1114}$ ) and sport, it can include other activities not traditionally thought of as exercise, such as gardening, carrying heavy shopping bags, or walking. When discussing physical activity with patients, reflect together on their goals and how they might achieve them. Understanding that small modifications count (such as carrying shopping home rather than driving) can help reshape perceptions of what is realistic, practical, and achievable.

\section{What are the risks of physical activity after covid-19?}

Current understanding of recovery from covid-19 is limited, but preliminary research has highlighted several key concerns. The first is the potential for cardiac injury, including from viral myocarditis (see box 1). This is important, as taking exercise in the presence of myocarditis is associated with increased morbidity and mortality. ${ }^{15}$

\section{Box 1: Myocarditis, covid-19, and exercise}

Most data on cardiac injury after covid-19 illness is from patients who were hospitalised and cannot be extrapolated to those with mild illness. ${ }^{15}$ Indeed, the incidence of myocarditis in those who were asymptomatic or had mild to moderate disease is unknown. ${ }^{13}$

One study of serum troponin measurements and cardiovascular magnetic resonance imaging in unselected patients after a diagnosis of covid-19 demonstrated ongoing myocardial inflammation in $60 \%$ at a median time of 71 days from diagnosis. ${ }^{4}$ Although $33 \%$ of participants were classified as severely unwell and required hospitalisation, and $67 \%$ were recovering at home, it is unclear how the mildness or severity of illness in the latter group was established, and how the recruitment procedure avoided the potential risks of selection bias. It is also unclear as to whether the findings indicate myocarditis or cardiac injury from other causes, and how clinically important the findings are for long term health or morbidity.

Indeed, in an autopsy study of 21 patients who died from covid-19, only three (14\%) were found to have 
lymphocytic infiltrates suggestive of myocarditis. ${ }^{16}$ Moreover, viral myocarditis can be a feature of many circulating viruses, including influenza. ${ }^{17}$

Thromboembolic complications, such as pulmonary emboli, are also associated with covid-19. ${ }^{18-20}$ Long term effects on pulmonary function are not currently known, but data from the 2003 severe acute respiratory syndrome coronavirus (SARS-CoV) epidemic suggest persistent impairments in pulmonary function and exercise capacity in survivors. $^{21}$

Finally, primary psychiatric phenomena, such as psychosis, have been identified as a potential presenting feature of covid-19, ${ }^{22}$ and psychological sequelae after infection can include post-traumatic stress disorder, anxiety, and depression. ${ }^{23}$

Some of these potential risks, viral myocarditis in particular, understandably lead to caution when advising a return to physical activity or exercise after infection. Without evidence from robust studies to inform practice, all current guidance to date is based on consensus or expert opinion. A consensus statement from sports clinicians of the European Federation of Sports Medicine Associations from July 2020 recommends a review with a sports and exercise medicine physician after mild symptomatic infection, and investigations including echocardiography and lung function testing where cardiopulmonary symptoms were present. ${ }^{24}$ Guidance from the Netherlands Society of Cardiology states that, for those with systemic features including fever, electrocardiography testing should be considered before resumption of activity. ${ }^{25}$ However, the incidence of myocardial injury (box 1) or thromboembolic complications after mild or moderate covid-19 in the community is currently unknown but thought to be low. Therefore, a balance is needed between obstructing an already inactive population from undertaking physical activity at recommended levels beneficial for their health, and the potential risk of cardiac or other consequences for a small minority. There is no perfect solution given the current uncertainties and the varying availability of resources globally, such as cardiopulmonary investigations or dedicated sports and exercise medicine services. We advocate a pragmatic approach that enables a gradual return to physical activity while mitigating risks.

\section{How do I know if my patient can safely return to physical activity?}

A risk-stratification approach can help maximise safety and mitigate risks, and a number of factors need to be taken into account. First, is the person physically ready to return to activity? In the natural course of covid-19, deterioration signifying severe infection often occurs at around a week from symptom onset. Therefore, consensus agreement is that a return to exercise or sporting activity should only occur after an asymptomatic period of at least seven days, ${ }^{21242627}$ and it would be pragmatic to apply this to any strenuous physical activity (fig 1). English and Scottish Institute of Sport guidance suggests that, before re-initiation of sport for athletes, activities of daily living should be easily achievable and the person able to walk $500 \mathrm{~m}$ on the flat without feeling excessive fatigue or breathlessness. ${ }^{27}$ However, we recommend considering the person's pre-illness baseline, and tailoring guidance accordingly. Some may not have been able to walk $500 \mathrm{~m}$ without breathlessness before their covid-19 illness, and they should not be precluded from starting physical activity at a level tolerable for them (see fig 1 , phases 1 to 3 ). 


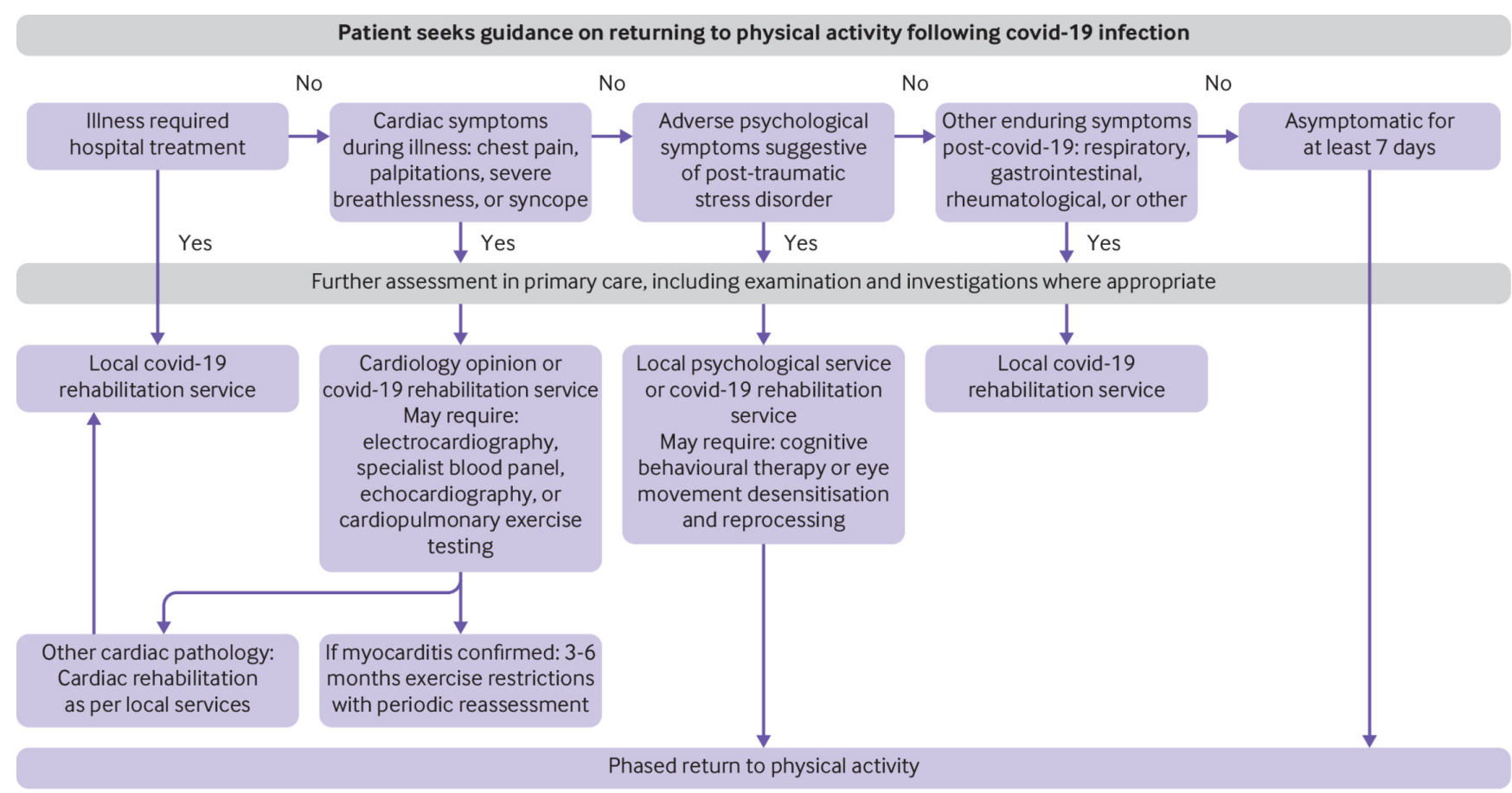

\begin{tabular}{|c|c|}
\hline Minimum of 7 days at eac & Drop back a \\
\hline $\begin{array}{l}\text { Phase } 1 \\
\text { Goal: preparation for return } \\
\text { to exercise } \\
\text { Exercise: rest, breathing } \\
\text { exercises, flexibility/ } \\
\text { stretching, balance, gentle } \\
\text { walking } \\
\text { Suggested Rating of } \\
\text { Perceived Exertion (RPE): } \\
\text { 6-8 }\end{array}$ & $\begin{array}{l}\text { Phase } 2 \\
\text { Goal: low intensity activity } \\
\text { such as walking and light } \\
\text { yoga, and light household/ } \\
\text { garden tasks } \\
\text { Exercise: graduated } \\
\text { increases by } 10-15 \text { mins/ } \\
\text { day } \\
\text { Suggested RPE: } 6-11 \\
\text { Progression: } 7 \text { days and } \\
\text { when can walk } 30 \\
\text { minutes at RPE } 11\end{array}$ \\
\hline
\end{tabular}

Phased return to physical activity

Fig 1 | Suggested return to physical activity after covid-19: risk stratification to exclude features suggestive of myocarditis or post-acute covid-19 and phased resumption of physical activity after 7 days without symptoms ${ }^{28}$

The second factor is that ongoing symptoms, regardless of system, may be indicative of a post-acute covid-19 illness. This will require assessment in primary care initially, and potentially liaison with local post-covid-19 rehabilitation services. ${ }^{121}$ Assessment and management of post-acute covid-19 illness is covered elsewhere. ${ }^{1}$ Whether there is a role for graded physical activity as a treatment for this condition is currently unclear.
People who had more severe covid-19 illness, such as those who were hospitalised, are thought to be at higher risk of cardiac complications ${ }^{121321242527}$ and thromboembolic events. ${ }^{1520}$ We recommend that their graduated rehabilitation be managed in conjunction, or after discussion and liaison, with local post-covid-19 services. People who did not require hospital treatment but who had symptoms during their illness suggestive of myocardial injury, such as chest pain, severe breathlessness, palpitations, symptoms 
or signs of heart failure, or syncope and pre-syncope, should be assessed with a physical examination and considered for further investigations. Depending on the severity of the symptoms encountered, investigations may include 12-lead electrocardiography, with abnormal findings prompting referral to cardiology or post-covid-19 services..$^{25}$ The cardio-respiratory examination may reveal signs suggestive of costochondritis or musculoskeletal pain, which can be safely managed in primary care. Discuss findings indicating pulmonary oedema, fibrosis, pleural effusion, added heart sounds, ongoing hypoxia, or new arrhythmias with local post-covid-19 rehabilitation or cardiology services. Investigations in secondary care may include serum troponin levels, electrocardiography, and echocardiography (fig 1)..$^{121325}$ Both European and US guidelines advocate restrictions on exercise for three to six months in cases of myocarditis confirmed by cardiac magnetic resonance imaging or endomyocardial biopsy. 1317212529

Third, with regards respiratory symptoms, persistent cough and breathlessness are expected to resolve after several weeks, ${ }^{115}$ but progressive, non-resolving or worsening symptoms may indicate pulmonary-vascular complications such as pulmonary embolism, concomitant pneumonia or post-inflammatory

bronchoconstriction, ${ }^{15}$ and these patients should be discussed with secondary care services accordingly.

Finally, is the person psychologically ready to embark on a physical activity programme? Physical activity has a positive impact on mood and mental wellbeing ${ }^{56}$ and has a role in the prevention and treatment of mental health conditions. The presence of such conditions should not preclude people from physical activity, but allows for additional support when needed. Psychological sequelae of covid-19 infection can be screened for in the consultation. Ask about mood, sleep, appetite, and motivation. Listen to,

acknowledge, and validate the patient's concerns. If further support is needed, people can be directed to self care resources, community services, and peer support. In some cases, coordination with local psychological support or post-covid-19 rehabilitation services may be needed (fig 1). ${ }^{121}$ Psychological screening tools for returning to physical activity and sport do exist, but they are likely to be outside the scope of the standard primary care consultation. ${ }^{27} 30$

\section{How do I guide a patient back to physical activity?}

There is no clear, evidence based way to guide return to physical activity, but a prudent approach is that it should be gradual, individualised, and based on subjective tolerance of the activity. Once a patient has been risk stratified and symptom-free for at least seven days, a phased approach can be used to increase physical activity levels to either baseline or guideline levels or beyond. ${ }^{21}$ If the person was not physically active before covid-19, this can be an opportune moment to discuss becoming more active. Return, or new development, of symptoms-including cough, abnormal breathlessness, palpitations, fever, and anosmia-indicate the need to stop, seek medical advice if required, and restart the process when symptom-free. ${ }^{27}$ People might be expected to be more breathless for a given activity after a period of illness or inactivity. However, a degree of subjective assessment is needed to assess if this is consistent with the given activity and level of fitness, and if it is improving. A graduated progression includes increases in volume (time doing the activity) and load (intensity).

Brief discussions regarding physical activity in primary care can follow a modified "3As" approach: ask, assess, and advise/assist. ${ }^{11}$ Begin by asking for permission to discuss the topic, and gauging the person's current levels of activity. Assess their current perspectives and goals regarding physical activity and if they would like to do more. Advise by providing information, such as that below, and signposting to resources (box 2). Suggest they set goals and consider monitoring their progress, such as by using a diary. Assist them by helping break down barriers (such as reinforcing that even brief periods of activity are effective for improving health, ${ }^{31}$ household and garden tasks all contribute, and that active travel is often a feasible way of incorporating physical activity into a day).

\section{Box 2: Key physical activity resources to guide patients}

- Department of Health and Social Care. UK chief medical officers' physical activity guidelines: infographics (https://www.gov.uk/government/publications/physical-activity-guidelines-infographics)

- Infographics with physical activity guidance across the lifespan, including for older adults, children, and during pregnancy/postpartum period

- Moving Medicine (https://movingmedicine.ac.uk/)

Online resource for healthcare professionals to help facilitate discussions with patients regarding physical activity

- Couch to 5k (https://www.nhs.uk/live-well/exercise/couch-to-5kweek-by-week/)

A graded programme to help people gradually ease into running

- OneYou (https://www.nhs.uk/oneyou/)

Tools and support to help guide and advise on physical activity

- Sport England. Join the movement (https://www.sportengland.org/jointhemovement)

- Physical activity advice and example workouts, including "Stay In Work Out" for exercise during lockdowns or when in isolation

- Chartered Society of Physiotherapists. Helping older people stay active at home (https://www.csp.org.uk/public-patient/keeping-active-and-healthy/staying-healthy-you-age/staying-strong-youage/strength)

- Simple exercises to help people, in particular older adults, to stay active at home

- We are undefeatable (https://weareundefeatable.co.uk/)

Guidance on being active for those with chronic health conditions

- Your COVID Recovery (https://www.yourcovidrecovery.nhs.uk/)

- Guidance on all steps on recovery from covid-19, including nutrition and physical activity

- COVID-19: Supporting your recovery (https://covidpatientsupport.lthtr.nhs.uk/\#/)

- Developed by Lancashire Teaching Hospitals, a resource with all aspects of rehabilitation after covid-19

- Chartered Society of Physiotherapists. Covid-19: the road to recovery (https://www.csp.org.uk/public-patient/covid-19-road-recovery)

Guidance on graduated return to physical activity after covid-19

- World Health Organization. Support for rehabilitation self-management after COVID-19-related illness (https://www.euro.who.int/en/healthtopics/Life-stages/disability-and-rehabilitation/publications/supportfor-rehabilitation-self-management-after-covid-19-related-illness2020-produced-by-whoeurope)

Advice for those who have been discharged from hospital with covid-19, including breathing exercises and physical activity 


\section{How to start}

\section{Phases 1-2}

Begin with light intensity activity for at least two weeks. ${ }^{13} 21$ The Borg Rating of Perceived Exertion (RPE) scale is a subjective assessment of how hard someone feels they are working and can be helpful to guide people in choosing what activities to do as they progress through the phases of increasing physical activity. They rate their complete subjective feeling of exertion, including shortness of breath and fatigue, on a scale from 6 (no exertion at all) to 20 (maximal exertion). ${ }^{28}$ Light intensity exercise is equivalent to an RPE of under 11 (fig 1), when a person feels minimal to light exertion. They should be able to hold a full conversation without difficulty at this level. Activities might include household and light garden tasks, gentle walking, and balance or yoga exercises. ${ }^{32}$ Breathing, stretching, and light strengthening activities can also be incorporated..$^{21}$ Examples of these can be found on the NHS website https://www.nhs.uk/live-well/exercise/flexibility-exercises/ and in the resources in box 2 . We recommend spending seven days (phase 1) on extremely light intensity activity (RPE 6-8), including flexibility and breathing exercises, for as long as the person feels able to do them, followed by a further seven days (phase 2) incorporating light intensity activity (RPE 6-11) such as walking and light yoga, with graduated increases at 10-15 minutes per day at the same RPE when tolerated.

\section{Phases 3-4}

Progress to more challenging movement activities depending on pre-illness capacity. These might include intervals of two 5-minute blocks of activity such as brisk walking, going up and down stairs, jogging, swimming, or cycling separated by a block of recovery. The person should not feel that the exercise is "hard," and we would suggest working to an RPE of 12-14 (moderate intensity, not out of breath and could hold a conversation). Progress by adding an interval per day as tolerated.

Phase 4 would involve more complex movement that challenges coordination, strength, and balance, such as running but with changes in direction, side-steps, shuffles, and circuits of body weight exercises, but again without it feeling hard. After completing phase 4 , people should then feel able to return to their baseline (pre-covid) level of activity or more.

We propose a minimum of seven days at each phase to prevent sudden increases in training load (see fig 1). However, people should stay at the phase they feel comfortable with for as long as necessary. They should monitor for any inability to feel recovered at 1 hour after exercise and on the day after, abnormal breathlessness, abnormal heart rate, excessive fatigue or lethargy, and markers of mental ill health. If these occur, or the person fails to progress as expected, they should step back to an earlier phase of activity and seek medical advice when unsure. Keeping a diary of exercise progression, along with RPE, any changes in mood, and, for those who are used to measuring it, objective fitness data such as heart rate, can be helpful for monitoring progress.

\section{A patient's perspective}

I work as a healthcare assistant and had my first symptoms of covid-19 in April, including shortness of breath, cough, and high heart-rate. Once the fever had ended, I felt extremely fatigued when doing the smallest amount of exercise. I was used to being able to walk and swim for at least an hour and a half, so this was debilitating for me

Using techniques from physiotherapy and the NHS covid patient support website, I slowly started to be able to do more. The exercise I found most helpful was stretching. This helped to expand my chest and lungs, so the more intense exercises got easier. It helped to be able to do these stretches prior to a more intense exercise such as walking, as my lungs felt they could hold more air. The breathing techniques were particularly helpful and something I do regularly. I found walking to also be most beneficial as it was an exercise I could control. I could walk at a speed and for a distance that was manageable for me and gradually increase this while using a "fitbit" to check my heart rate and recovery time.

It has now been about 10 weeks since my first symptoms, and I would say I am at around $70 \%$ of my pre-covid fitness. I am aware of my own limitations and am still working to improve these every day.

\section{Social inequality, physical inactivity, and covid-19}

Levels of physical activity vary substantially across society in the UK: women, people from lower socio-economic backgrounds, and those from some ethnic minorities are less likely to be physically active. ${ }^{8} 33$ Multiple factors contribute, including cultural acceptance, prevailing concepts of gender, 34 relative cost, time, access to childcare and awareness of available opportunities for physical activity or exercise. ${ }^{35}$ A qualitative synthesis study of barriers to physical activity in individuals from black and minority ethnic groups ${ }^{36}$ showed varying concepts of physical activity shaped by socio-cultural factors, and of failures to engage with such individuals at an organisational level. Inequalities in physical activity have impacts across a whole community: unequal distribution of physical activity across a country seems to be associated with an increased prevalence of obesity, to a greater extent than from average levels of activity. 37

Although many of the solutions are at a structural and policy level, 33 there is also a key role for physical activity promotion in primary care. We suggest:

- Brief and opportunistic intervenions, ${ }^{11}$ such as when reviewing a patient after covid-19 illness or when discussing long term disease management. Use the $3 \mathrm{~A}$ framework described in the article to introduce the topic into consultations. The Moving Medicine resource (see box 2) can guide conversations on physical activity. The UK chief medical officers' physical activity guidelines infographics (box 2) can be printed for patients to take home.

- Understanding influences and barriers to physical activity for the patient may inform discussion. For cost, many local leisure services offer discounted access; for time, active travel or commuting can be part of a solution. Social prescribing link workers or navigators can help people access the community services available to them. Fitness and activity opportunities are also included as an option on the service finder page of the NHS website (https://www.nhs.uk/servicesearch/other-services/Fitness-activities-and-classes/LocationSearch/671).

- Consider making your practice an "Active Practice": the Royal College of General Practitioners offers advice on this in their Physical Activity and Lifestyle Toolkit (https://www.rcgp.org.uk/clinical-and-research/resources/toolkits/physical-activity-and-lifestyle.aspx). Examples of practice-level changes include displaying physical activity guidelines on the right hand side of $\mathrm{FP}_{10}$ prescriptions, sending targeted exercise advice to groups such as those with diabetes or hypertension, and displaying information on local physical activity resources within the practice or on the practice website.

\section{How this article was created}

We performed a literature search using Ovid, and searched the Medline, Embase, and Global Health databases using the search terms (physical activity OR exercise OR sport\$ OR rehab\$) AND (coronavirus OR covid 19 OR post-covid\$)). Articles from 2019-20 were screened. We also searched for consensus statements and guidelines, including those from the British Association of Sports and Exercise Medicine (BASEM), British Thoracic Society (BTS), Chartered Society of Physiotherapy (CSP), English Institute of Sport (EIS), British Society of Rehabilitation Medicine (BSRM), European Society of Cardiology, and American College of Sports Medicine (ACSM). 


\section{Education into practice}

- How often do you feel able to recommend physical activity guidance in clinical practice?

- How do you account for social inequalities when discussing lifestyle factors, such as physical activity or diet, in your practice?

\section{How patients were involved in the creation of this article}

We thank the patient who provided their personal story of their illness and recovery, and how this affected their return to physical activity. Their recollection, together with those of several other patients, created the impetus for writing this guidance, emphasised the importance of this work, and guided its development.

Contributors: DS, DV, and AHM conceived the article and are guarantors. All authors wrote and reviewed the article, created the boxes, and helped with the figures. PLF was the contact for patient involvement.

Funding: This article was in part supported by the National Institute for Health Research Applied Research Collaboration North west London. TB and DS are supported by Health Education England / National Institute for Health Research (NIHR) Academic Clinical Fellowships. TB acknowledges support from the NIHR Imperial Biomedical Research Centre (BRC). The views expressed in this publication are those of the authors and not necessarily those of Health Education England, the National Institute for Health Research or the Department of Health and Social Care.

Competing interests: We have read and understood the BMJ Group policy on declaration of interests and have no relevant interests to declare.

We thank Move Imperial for their help in the early stages of this project, when this guidance played a role in guiding scholarship athletes at Imperial College back to training. We are also grateful for the comments and feedback from rehabilitation clinicians at the Defence Medical Rehabilitation Centre (DMRC) at Stanford Hall. We thank the patient who kindly contributed and revised her personal story.

1 Greenhalgh T, Knight M, A'Court C, Buxton M, Husain L. Management of post-acute covid-19 in primary care. BMJ2020;370:m3026. doi: 10.1136/bmj.m3026 pmid: 32784198

2 Carfi A, Bernabei R, Landi FGemelli Against COVID-19 Post-Acute Care Study Group. Persistent symptoms in patients after acute COVID-19. JAMA 2020;324:603-5.

doi: 10.1001/jama.2020.12603. pmid: 32644129

3 Pan F, Ye T, Sun P, etal. Time course of lung changes on chest CT during recovery from 2019 novel coronavirus (COVID-19) pneumonia. Radiology 2020;295:200370doi: 10.1148/radiol.2020200370

4 Puntmann VO, Carerj ML, Wieters I, etal. Outcomes of cardiovascular magnetic resonance imaging in patients recently recovered from coronavirus disease 2019 (COVID-19). JAMA Cardiol 2020;5:1265-73. doi: 10.1001/jamacardio.2020.3557. pmid: 32730619

5 Public Health England. Health matters: getting every adult active every day. Gov.co.uk. 2016. https://www.gov.uk/government/publications/health-matters-getting-every-adult-active-everyday.

6 Department of Health and Social Care. Physical activity guidelines: UK Chief Medical Officers' report. 2019. https://www.gov.uk/government/publications/physical-activity-guidelines-uk-chiefmedical-officers-report.

7 Lee I-MM, Shiroma EJ, Lobelo F, Puska P, Blair SN, Katzmarzyk PTLancet Physical Activity Series Working Group. Effect of physical inactivity on major non-communicable diseases worldwide: an analysis of burden of disease and life expectancy. Lancet 2012;380:219-29. doi: 10.1016/S0140-6736(12)61031-9 pmid: 22818936

8 Sport England. Active lives: adult survey november 2018/19 report. 2020. https://sportenglandproduction-files.s3.eu-west-2.amazonaws.com/s3fs-public/2020-04/Active\%20Lives\%20Adult\%20November\%2018-19\%20Report.pdf?BhkAy2K28pd9bDEz_NuisHI2ppuq|tpZ.

9 Rogers NT, Waterlow NR, Brindle H, etal. Behavioral change towards reduced intensity physical activity is disproportionately prevalent among adults with serious health issues or self-perception of high risk during the UK COVID-19 lockdown. Front Public Health 2020;8:575091. doi: 10.3389/fpubh.2020.575091 pmid: 33102424

10 Sheldon TA, Wright J. Twin epidemics of covid-19 and non-communicable disease. BMJ 2020;369:m2618. doi: 10.1136/bmj.m2618 pmid: 32605906

11 Haseler C, Crooke R, Haseler T. Promoting physical activity to patients. BM/2019;366:15230 doi: 10.1136/bmj.15230 pmid: 31530549

12 Bhatia RT, Marwaha S, Malhotra A, etal. Exercise in the severe acute respiratory syndrome coronavirus-2 (SARS-CoV-2) era: A question and answer session with the experts endorsed by the section of sports cardiology \& exercise of the European Association of Preventive Cardiology (EAPC). Eur J Prev Cardiol2020;27:1242-51. doi: 10.1177/2047487320930596 pmid: 32475157

13 Phelan D, Kim JH, Chung EH. A game plan for the resumption of sport and exercise after coronavirus disease 2019 (COVID-19) infection. JAMA Cardiol 2020;

doi: 10.1001/jamacardio.2020.2136. pmid: 32402054
14 Caspersen CI, Powell KE, Christenson GM. Physical activity, exercise, and physical fitness: definitions and distinctions for health-related research. Public Health Rep 1985;100:126-31.pmid: 3920711

15 Wilson MG, Hull JH, Rogers J, etal. Cardiorespiratory considerations for return-to-play in elite athletes after COVID-19 infection: a practical guide for sport and exercise medicine physicians. Br J Sports Med 2020;54:1157-61. doi: 10.1136/bjsports-2020-102710 pmid: 32878870

16 Basso C, Leone O, Rizzo S, etal. Pathological features of COVID-19-associated myocardial injury: a multicentre cardiovascular pathology study. Eur Heart J2020;41:3827-35. doi: 10.1093/eurhearti/ehaa664 pmid: 32968776

17 Caforio ALP, Pankuweit S, Arbustini E, etalEuropean Society of Cardiology Working Group on Myocardial and Pericardial Diseases. Current state of knowledge on aetiology, diagnosis, management, and therapy of myocarditis: a position statement of the European Society of Cardiology Working Group on Myocardial and Pericardial Diseases. Eur Heart J2013;34:2636-48, 2648a-2648d. doi: 10.1093/eurheartj/eht210 pmid: 23824828

18 Gupta A, Madhavan MV, Sehgal K, etal. Extrapulmonary manifestations of COVID-19. Nat Med 2020;26:1017-32. doi: 10.1038/s41591-020-0968-3 pmid: 32651579

19 Ackermann M, Verleden SE, Kuehnel M, etal. Pulmonary vascular endothelialitis, thrombosis, and angiogenesis in Covid-19. N Engl / Med 2020;383:120-8. doi: 10.1056/NEJMoa2015432 pmid: 32437596

20 Bompard F, Monnier H, Saab I, etal. Pulmonary embolism in patients with COVID-19 pneumonia Eur Respir J2020;56:2001365. doi: 10.1183/13993003.01365-2020 pmid: 32398297

21 Barker-Davies RM, O'Sullivan O, Senaratne KPP, etal. The Stanford Hall consensus statement for post-COVID-19 rehabilitation. Br J Sports Med 2020;54:949-59. doi: 10.1136/bjsports-2020-102596. pmid: 32475821

22 Varatharaj A, Thomas N, Ellul MA, etalCoroNerve Study Group. Neurological and neuropsychiatric complications of COVID-19 in 153 patients: a UK-wide surveillance study. Lancet Psychiatry 2020;7:875-82. doi: 10.1016/S2215-0366(20)30287-X pmid: 32593341

23 Mazza MG, De Lorenzo R, Conte C, etalCOVID-19 BioB Outpatient Clinic Study group. Anxiety and depression in COVID-19 survivors: Role of inflammatory and clinical predictors. Brain Behav Immun 2020;89:594-600. doi: 10.1016/j.bbi.2020.07.037 pmid: 32738287

24 Löllgen H, Bachl N, Papadopoulou T, etal. Recommendations for return to sport during the SARS-CoV-2 pandemic. BMJ Open Sport Exerc Med 2020;6:e000858doi: 10.1136/bmisem-2020-000858.

25 Verwoert GC, de Vries ST, Bijsterveld N, etal. Return to sports after COVID-19: a position paper from the Dutch Sports Cardiology Section of the Netherlands Society of Cardiology. Neth Heart J2020;28:391-5. doi: 10.1007/s12471-020-01469-z pmid: 32662058

26 Hull JH, Loosemore M, Schwellnus M. Respiratory health in athletes: facing the COVID-19 challenge Lancet Respir Med 2020;8:557-8. doi: 10.1016/S2213-2600(20)30175-2. pmid: 32277869

27 Elliott N, Martin R, Heron N, Elliott J, Grimstead D, Biswas A. Infographic. Graduated return to play guidance following COVID-19 infection. Br J Sports Med 2020;54:1174-5. doi: 10.1136/bjsports-2020-102637 pmid: 32571796

28 Williams N. The Borg Rating of Perceived Exertion (RPE) scale. Occup Med (Chic III) 2017;67:404-5doi: 10.1093/occmed/kqx063.

29 Dores H, Cardim N. Return to play after COVID-19: a sport cardiologist's view. Br J Sports Med 2020;54:1132-3. doi: 10.1136/bjsports-2020-102482. pmid: 32381502

30 Glazer DD. Development and preliminary validation of the Injury-Psychological Readiness to Return to Sport (I-PRRS) scale. J Athl Train 2009:44:185-9. doi: 10.4085/1062-6050-44.2.185. pmid: 19295964

31 Saint-Maurice PF, Troiano RP, Matthews CE, Kraus WE. Moderate-to-vigorous physical activity and all-cause mortality: do bouts matter?) Am Heart Assoc 2018;7:e007678. doi: 10.1161/JAHA.117.007678. pmid: 29567764

32 Ainsworth BE, Haskell WL, Herrmann SD, etal. 2011 Compendium of Physical Activities: a second update of codes and MET values. Med Sci Sports Exerc 2011;43:1575-81. doi: 10.1249/MSS.0b013e31821ece12. pmid: 21681120

33 British Medical Association. Get a move on: Steps to increase physical activity levels in the UK. 2019. https://www.bma.org.uk/media/2104/bma-physical-activity-briefing-get-move-on-oct-19. V2.pdf.

34 The Lancet Public Health. Time to tackle the physical activity gender gap. Lancet Public Health 2019;4:e360. doi: 10.1016/S2468-2667(19)30135-5 pmid: 31345750

35 Withall J, Jago R, Fox KR. Why some do but most don't. Barriers and enablers to engaging low-income groups in physical activity programmes: a mixed methods study. BMC Public Health 2011;11:507. doi: 10.1186/1471-2458-11-507 pmid: 21711514

36 Koshoedo SA, Paul-Ebhohimhen VA, Jepson RG, Watson MC. Understanding the complex interplay of barriers to physical activity amongst black and minority ethnic groups in the United Kingdom: a qualitative synthesis using meta-ethnography. BMC Public Health 2015;15:643. doi: 10.1186/s12889-015-1893-0 pmid: 26164652

37 Althoff T, Sosič R, Hicks JL, King AC, Delp SL, Leskovec J. Large-scale physical activity data reveal worldwide activity inequality. Nature2017;547:336-9. doi: 10.1038/nature23018 pmid: 28693034 